\title{
Difficult Airway Management in a Child with Goldenhar Syndrome: A Case Report
}

\author{
Malaka Munasinghe ${ }^{1 *}$, Nishanthan Subramaniam ${ }^{2}$, Nimalan Srisothinathan ${ }^{3}$, Binoy Ranatunga ${ }^{4}$, \\ Kasun Ranaweera ${ }^{5}$, Charith Rathuwithana ${ }^{6}$, Ajith Appuhamy ${ }^{7}$ \\ ${ }^{1}$ Acting Consultant Anaesthetist, ${ }^{2,3,4,5}$ Medical officer in Anaesthesia and Intensive care,${ }^{6}$ Acting \\ Consultant Paediatrician, ${ }^{7}$ Consultant Orthopaedic Surgeon, District General Hospital, Mannar, \\ Sri Lanka
}

\begin{abstract}
Goldenhar Syndrome is a rare congenital entity which comprises of facial dysmorphism, oral cavity malformations and vertebral anomalies any of which could lead to difficult airway during anaesthesia. Here, we report a case of a 5-month-old infant who underwent a congenital talipes equinovarus (CTEV) correction under general anaesthesia. We experienced difficulty in mask ventilation, failed endotracheal intubation and transient desaturation which was anticipatedthus managed successfully without serious morbidity to the patient.
\end{abstract}

Key Words: Goldenhar syndrome, difficult airway, hemifacial hypoplasia

\section{Case Report}

A 5-month-old, $6 \mathrm{~kg}$ infant delivered at term and diagnosed with Goldenhar syndrome was planned for a right side CTEV correction. During the preoperative assessment the following were noticed; pre-auricular skin tags, right eye coloboma, congenital atrial septal defect (ASD) and absent kidney on the right side. There was no history suggestive of upper airway obstruction. The airway assessment revealed micrognathia (Fig. 1 and 2).

There were no palatal or vertebral anomalies. Schwartz hyoid maneuver and Colorado Paediatric Airway Scores (COPUR) were $1.2 \mathrm{~cm}$ and 10 respectively, both predicting difficult airway. Cardiac assessment and renal functions were normal. There was no past history of anaesthesia, any chronic illness or allergies. The infant was kept fasted in accordance with local protocols.

*Correspondence: Malaka Munasinghe E mail: malakafmp@gmail.com

https://orcid.org/0000-0001-8373-4752

Received: 30/07/2020

Accepted: $11 / 09 / 2020$

DOI: http:/doi.org/10.4038/slja.v29i1.8648

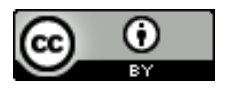

Paracetamol $90 \mathrm{mg}$ was given 1-hour prior as preemptive analgesia. Informed written consent was taken from the parents following a discussion on the possibility of difficult airway and emergent tracheostomy.

With anticipated difficulty, airway cart consisting of adequately sized ET tubes, straight and curved laryngoscope blades, paediatric bougie and oropharyngeal airways with laryngeal mask airway (LMA) were kept ready. Videolaryngoscope and intubating laryngeal mask were

\section{Figure 1}

Lateral view of the head and face; (M) Micrognathia, (P) - Pre-auricular skin tag

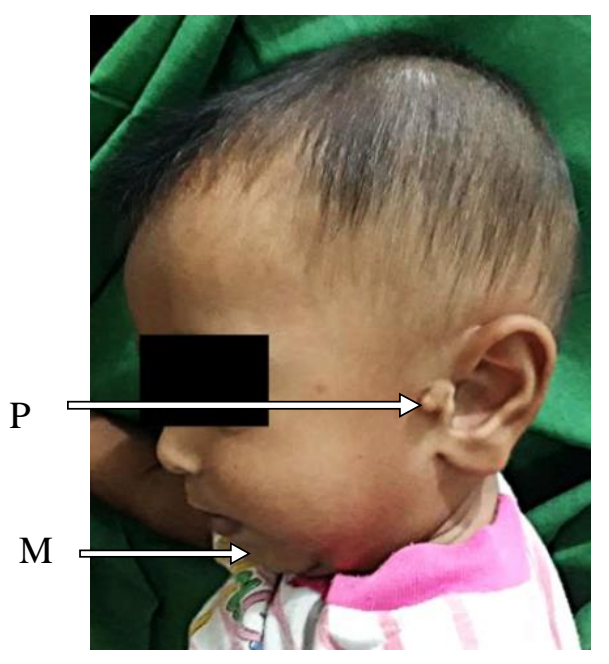




\section{Figure 2}

X-Ray head and neck (lateral view); excludes atlanto-axial subluxation

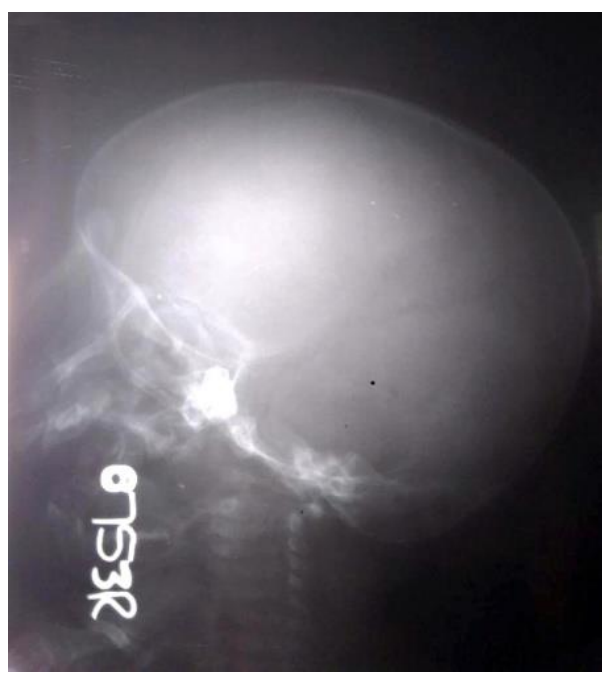

not available at our institution. Awake fiber-optic intubation was considered impractical due to the child's age. Consultant ENT surgeon was present inside the theatre during intubation with emergency tracheostomy set.

Expected surgical time was around 3 hours, with probable need for lateral positioning. For positional security of the airway and to prevent gastric insufflation hindering pulmonary compliance, intubation was preferred over LMA. Standard AAGBI monitoring was commenced. Patient was pre-oxygenated with $100 \%$ oxygen and induced with sevoflurane. With adequate depth of anaesthesia and optimum position, intubation was attempted with size 1 straight blade. Two attempts at intubation failed with a laryngoscopic view (Cormack and Lehane grading) of 4. Oxygenation was maintained in between attempts. During the second attempt patient desaturated to $85 \%$. There was no haemodynamic instability, laryngospasm, bronchospasm or pneumothorax. Assisted face mask ventilation with two hand technique was also found to be difficult. Subsequently, an oropharyngeal airway was placed and patient's spontaneous ventilation was successfully assisted. Following adequate oxygenation and adequate depth of anaesthesia, air way was secured with a size 1 classic LMA. Anaesthesia was maintained with $\mathrm{N}_{2} \mathrm{O}: \mathrm{O}_{2}$ : isoflurane $1 \%$ with spontaneous ventilation. Caudal block was administered and surgery was conducted in supine position. At the end of a 2-hour surgery, LMA was removed once patient was fully awake at left lateral position. The child was discharged the next day after an uneventful post-operative period.

\section{Discussion}

While anaesthetizing a child is challenging, anaesthetizing syndromic children is further complicated by airway, cardiac, musculoskeletal and neurological anomalies.

The cranio-facial anomalies such as hemifacial hypoplasia are common in Goldenhar syndrome ${ }^{1}$ thus can lead to difficulty in ventilation. Absent or fused ribs and vertebral anomalies, e.g.; scoliosis, may cause reduced safe period of apnoea. Similarly, concomitant cleft lip, cleft palate, temporomandibular joint anomalies and cervical vertebral anomalies like atlanto-axial subluxation could also lead to difficult airway. Obstructive sleep apnoea should also be sought in history. The scoring systems utilized to assess adult airways are less sensitive and specific in paediatric population. The Schwartz hyoid maneuver which measures the anteroposterior distance from the middle of the inside of the mentum of the mandible to the hyoid bone could be useful in assessing potential space for laryngoscopy ${ }^{3}(>1.3 \mathrm{~cm}$ is considered adequate in infants). Colorado Paediatric Airway Score (COPUR) is another airway assessment tool used by some ${ }^{2}$ (a cumulative score $>10$, predicts difficult airway). The associated cardiac anomalies could result in haemodynamic instability during induction which complicates this crucial period of anaesthesia. $^{4}$

Although some clinicians prefer gaseous induction while maintaining spontaneous ventilation in view of predicted difficult airway in this cohort ${ }^{5}$,Madan et al, reported successful intravenous induction and muscle paralysis adopted during intubation. ${ }^{3}$ Awake fiber-optic intubation is an alternative but practically quite difficult in this age group due to unavailability of the equipment with appropriate size and uncooperative child etc. Use of video laryngoscope is becoming popular among anaesthetists with its superior quality of airway visualization. ${ }^{6}$ Use of optical stylets, intubation fibroscopically using intubating LMA are other 
documented methods of airway control. ${ }^{7,8,9}$ Retrograde intubation via a catheter guided through cricothyroid membrane and subsequent intubation is also reported as a method of securing airway ${ }^{10}$. Surgical tracheostomy has been required in few documented cases thus it is wise to have stand-by assistance of an ENT surgeon. It is also of paramount importance to maintain oxygenation as cardiac instability could follow episodes of desaturation.

Due to the unavailability of advanced airway equipment, we planned ahead the management of airway and were prepared for difficulty in ventilation or intubation. Even though, intubation failed, which was followed by transient desaturation, we succeeded in placing an LMA and proceeding with surgery without further instability.

Apnoeic spells have been reported during the postoperative period specially among infants thus vigilance should be continued throughout this period as well.

The associated vertebral anomalies could lead to difficult regional anaesthetic techniques like caudal epidural blocks. Depending on the clinical examination, radiological clarification using $\mathrm{x}$-ray (x-ray spine, skull etc.) or CT/MRI might be required in some patients before anaesthesia.

\section{Conclusion}

Provision of anaesthesia for children with Goldenhar syndrome needs careful planning at each step of anaesthesia and should be tailored to each. The management of airway could be problematic and lead to catastrophes thus scrupulous attention is required with advanced airway equipment and expert help.

Conflicts of interest - None declared by authors. Informed written consent was obtained from the parents for publishing the photos and clinical details.

\section{References}

1. Carey JC. Syndromes of the head and neck. Robert J. Gorlin, M. Michael Cohen, Jr., and L. Stefan Levin. New York: Oxford University Press, 1990, 977 pp. Am J Med Genet. 1992; 42(1):144-144. https://doi:10.1002/ajmg.1320420133
2. Raj D, Luginbuehl I. Managing the difficult airway in the syndromic child. Contin Educ Anaesthesia, Crit Care Pain. 2015; 15 (1): 7-13. https://doi:10.1093/bjaceaccp/mku004

3. Madan R, Trikha A, Venkataraman RKet al. Goldenhar's syndrome: an analysis of anaesthetic management A retrospective study of seventeen cases. Anaesthesia. 1990; 45 (1): 49-52. https://doi:10.1111/j.1365-2044.1990.tb14505.x PMID: 2316841

4. Choudhury M, Kapoor P. Goldenhar syndrome: Cardiac anesthesiologist's perspective. Ann Card Anaesth. 2017; 20 (5): S61-S66. https://doi:10.4103/0971-9784.197802 PMID: 28074825 PMCID: PMC5299831

5. Roodneshin F, Agah M. Management of anesthesia in Goldenhar syndrome: case-series study. Tanaffos. 2009; 8: 43-50

6. Kajal S, Mukherjee S, Saxena A, Asthana U. Airway management in a patient with Goldenhar syndrome. Sri Lankan J Anaesthesiol. 2017; 25 (1): 44.

https://doi:10.4038/slja.v25i1.8179

7. Chen PP, Cheng CK, Abdullah V, Chu CPW. Tracheal intubation using suspension laryngoscopy in an infant with Goldenhar's syndrome. Anaesth Intensive Care. 2001; 29 (5): 548-551. https://doi:10.1177/0310057x0102900519 PMID: 11669441

8. Okuyama M, Imai M, Fujisawa Eet al. Fiberscopic intubation under general anesthesia for children with Goldenhar syndrome. Masui.1994; 43 (12): 1885-8. PMID:7837410

9. Haxby EJ, Liban JB. Fibreoptic intubation via a laryngeal mask in an infant with Goldenhar syndrome. Anaesth Intensive Care. 1995; 23 (6): 753.

PMID: 8669621

10. Hasani R, Shetty A, Shinde S. Retrograde intubation: a rare case of goldenhar syndrome posted for posterior fossa surgery in the sitting position. Journal of Neurosurgical Anesthesiology. 2013 Oct; 25 (4): 428. https://doi: 10.1097/ana.0b013e318294f26a. PMID: 23715043 